\title{
Segmentation of Pathological Heart Sound Signal Using Empirical Mode Decomposition
}

\author{
Daoud Boutana, Braham Barkat, and Messaoud Benidir
}

\begin{abstract}
The Phonocardiogram (PCG) is the graphical recording of acoustic energy produced by the mechanical activity of various cardiac. Due to the complicated mechanisms involved in the generation of in the PCG signal, it is considered as multicomponent non stationary signal. Empirical mode decomposition (EMD) allows decomposing an observed multicomponent signal into a set of monocomponent signals, called Intrinsic Mode Functions (IMFs). The goal of this paper is to segment some pathological HS signals into the murmurs related to cardiac diseases. EMD approach allows to automatically selecting the most appropriate IMFs characterizing the murmur using the noise only model. Real-life signals are used in the various cases such as Early Aortic Stenosis (EAS), Late Aortic Stenosis (LAS), Mitral Regurgitation (MR) and Aortic Regurgitation (AR) to validate, and demonstrate the effectiveness of the proposed method.
\end{abstract}

Index Terms-Empirical mode decomposition, heart sound signal, pathological murmurs, noise only model.

\section{INTRODUCTION}

Heart sound segmentation is considered as a helpful operation for detection and partitioning in main component and especially pathological murmur in each cardiac cycle. The two main audible Heart Sounds (HS) in a normal cardiac cycle are the first (S1) and second (S2) heart sound. The first heart sound $\mathrm{S} 1$ consists of two major components $\mathrm{M} 1$ and T1, corresponding, respectively to the mitral and tricuspid valves. However the second heart sound S2 consists of two main components noted $\mathrm{A} 2$ and $\mathrm{P} 2$ corresponding respectively to the aortic and pulmonary valves. The empirical mode decomposition (EMD) method was first proposed by Huang et al. in 1998 [1]. For a nonstationary multicomponent signal, the EMD technique is a powerful tool and permits the decomposition on mono- components such as amplitude and frequency modulated (AM/FM) called the intrinsic mode functions (IMFs). In particular, the EMD technique expands the analyzed signal in terms of basic functions that are signal-dependent, and which can be estimated via an iterative procedure called sifting. The EMD method has been studied with an efficient algorithm for its implementation has been presented [2]. The method has also been used for denoising and detrending signals corrupted with noise $[3,4,5]$. EMD approach has also been used in denoising and time frequency

Manuscript received October 9, 2012; revised November 28, 2012

D. Boutana is with the Department of Automatic, faculty of science and technology, university of Jijel Algeria (e-mail daoud.boutana@mail.com).

B. Barkat is with the Department of Electrical Engineering, The petroleum Institute, Abu Dhabi, United Arab Emirates (e-mail: bbarkat@pi.ac.ae ).

M. Benidir is with the Laboratoire des signaux et systèmes, supelec, université Paris-Sud, 91192 Gif sur-Yvette, France (e-mail benidir@LSS.supelec.fr ). caracterisation of normal heart sound signal in [6].The problem of HS signal segmentation have been studied [7] using normalized average Shannon energy to compute the PCG envelope.

Another paper present a successfully method for HS segmentation [8] based on Shannon energy. However, the method fails to segment heart pathological murmurs. Samjin Choi et al. [9] used the normalized average Shannon energy, the envelope information of Hilbert transform with cardiac sound characteristic waveform in order to extract the envelope for the cardiac sound signal segmentation. The authors in [10] proposed a new method of HS biometrics using a synthetic model of HS based on the wavelet family. Recently, Boutana et al in [11] used the time frequency distribution with Rényi entropy measure for identification and segmentation of some pathological HS sound signals. A study of the characteristics of white noise using the EMD method has been presented [12].The EMD method has also been used for denoising and detrending signals using the noise-only model [13].

The paper is organized as follows. In Section II, we present an overview of the EMD method. In Section III, we present the noise only model as segmentation method. Section IV presents various pathological cases and experimental results, and Section $\mathrm{V}$ summarizes and concludes the paper.

\section{BACKGRoud of EMPIRICAL Mode DeCOMPOSITION}

The EMD is a signal decomposition method that allows the separation of the observed signal into a set of IMFs plus a residual function. Each IMF represents a monocomponent signal or an oscillatory mode with one instantaneous frequency. An IMF must satisfy the following two basic conditions [1]:

1) The number of extrema and the number of zero crossings must be either equal or different by no more than one in the entire data set,

2) The mean value of the envelope defined by the local maxima and the envelope defined by local minima is zero at the energy point. Thus, an IMF represents a simple oscillatory mode imbedded in the signal.

To extract an IMF from a given signal, we proceed according to the following EMD algorithm [1]:

Step 1: Identify the extrema (local maxima and minima) of the observed signal $x(t)$.

Step 2: Interpolate the local extrema using cubic spline to obtain the upper and the lower envelopes.

Step 3: Calculate the local mean value $m_{l}(t)$ of the upper and the lower envelopes.

Step 4: Subtract $m_{l}(t)$ from the original signal to obtain the 
first component $h_{1}(t)=x(t)-m_{1}(t)$

Step 5: If $h_{l}(t)$ satisfies the two basic properties mentioned in points (a) and (b) above, then, declare it as an IMF.

Step 6: if not, $h_{l}(t)$ is considered as a new original signal, and the above steps are repeated until we obtain an IMF, noted as $C_{I}(t)$. At this stage, we obtain the residual function $r_{l}(t)$ as:

$$
r_{1}(t)=x(t)-C_{1}(t)
$$

To obtain the next IMF, $r_{l}(t)$ is now considered as a new signal and the above steps are repeated again. At the end of the algorithm, we obtain a set of IMFs and a residue, whereby, the first extracted IMF contains the highest frequency oscillation that exists in the signal. Each extracted IMF contains a lower frequency oscillation than the one extracted just before it. We observe that the residual function is constant or monotonic function without frequency components. Finally, we can express the original signal $x(t)$ as follows:

$$
x(t)=\sum_{i=1}^{n} C_{i}(t)+r_{n}(t)
$$

where $C_{i}(t)$ represents the extracted IMFs and $r_{n}(t)$ is the residual function.

\section{Noise Only Model AS Segmentation Method}

Current methods to select the IMFs are based on a technique called noise-only model. The noise-only model technique uses the fact that in the presence of additive white Gaussian noise, the logarithm-variance of each IMF varies linearly with a parameter, called the Hurst exponent $H$ [12], [13] as follows:

$$
\log _{2} V_{H}(i)=\log _{2} V_{H}(2)+2(H-1)(i-2) \log _{2} \rho_{H}
$$

For $i \geq 2$ and $\rho_{\mathrm{H}}=2.01$ with $V_{H}(i)$ is the variance of the ith IMF. For this model used in [3]-[5], the IMF energies can be estimated using the equation:

$$
\hat{E}_{i}=\frac{\hat{\sigma}_{n}^{2}}{0.719} 2.01^{-i} \quad, i=2,3,4, \ldots \ldots \ldots
$$

where $\hat{\sigma}_{n}^{2}$ represent the energy of noise and may be estimated by the variance of the first IMF. Thus, after applying the EMD algorithm, an extracted signal component may be identified as an IMF of the signal containing the main components if its logarithm-variance verifies the linearity property and that energy exceed the threshold given by the noise-only model. In this paper, our motivation is to discriminate the IMFs corresponding to the murmur from the set of all extracted ones. The estimation of IMFs energy provides two situations: (i) if the IMF has energy greater than the threshold, then the IMF correspond to the signal without murmur, (ii) if the IMF has energy less than the threshold, then the IMF may be considered as murmur signal.

\section{EXPERIMENTAL RESUlTS AND DISCUSSION}

The segmentation study was conducted on abnormal real-life PCG signals collected from [14].Each PCG signal was sampled at a frequency: EAS and LAS signals with 11 $\mathrm{KHz}, \mathrm{MR}$ and ARsignals with $8 \mathrm{KHz}$.

\section{A. Example 1: Case of Early Aortic Stenosis}

In the first example, the method is applied on the PCG signal of a patient affected by Early Aortic Stenosis (EAS) showing a systolic murmur between the signal S1 and S2 such as in the top of the Fig. 1 EMD analysis of the EAS signal has revealed sixteen IMFs and a residue showed in the Fig. 1 Obviously, it can be seen that the third first IMF contains the most part of the murmur. However, the main components of the signal appear from the fourth IMF. According to the Fig. 2, it can be seen that between all extracted IMFs, the IMF1,IMF2 and IMF3 are principaly the only ones with energy not exceeds the threshold and may be selected as the IMFs for the separated murmur caracterising the EAS signal.The applied method has given the segmentation results illustrated by the Fig. 3 and show a best segmentation of the main components and the murmur of the EAS signal.

\section{B. Example 2: Case of Late Aortic Stenosis}

In the case of Late Aortic Stenosis (LAS), the pathological murmur is located in late systole just before the start of the second heart sound signal (S2). The applied method to the signal has given $18 \mathrm{IMFs}$ and a residue. In order to illustrate clear results, we have presented only 12 IMFs and the residue in the Fig. 4. The IMFs selection results are illustrated in Fig. 5 . It can be seen that the murmur is represented by the first third IMFs. The segmentation results are showed by the Fig. 6 where the parts (b) and (c) give the separated signal containing the main components and murmur respectively.

\section{Example 3: Case of Mitral Regurgitation}

The other pathological HS signal used in order to validate the method is the Mitral Regurgitation (MR), sometimes called mitral insufficiency. In the case of the MR, the valve does not close properly and causes blood to leak back (regurgitate) into the left atrium when the left ventricle contracts. MR is mainly characterized by systolic murmurs starting just after the first HS noted S1 and the presence of the third HS noted $\mathrm{S} 3$ as can be seen in the top of the Fig. 7 EMD analysis of the MR signal has revealed nine IMFs and a residue showed in the Fig.7. It can be seen in Fig. 8 and 9 that the third first IMF contains the most part of the murmur. So, the summation of the first third IMFs has given the separated murmur as can be seen in the Fig. 9 (c).

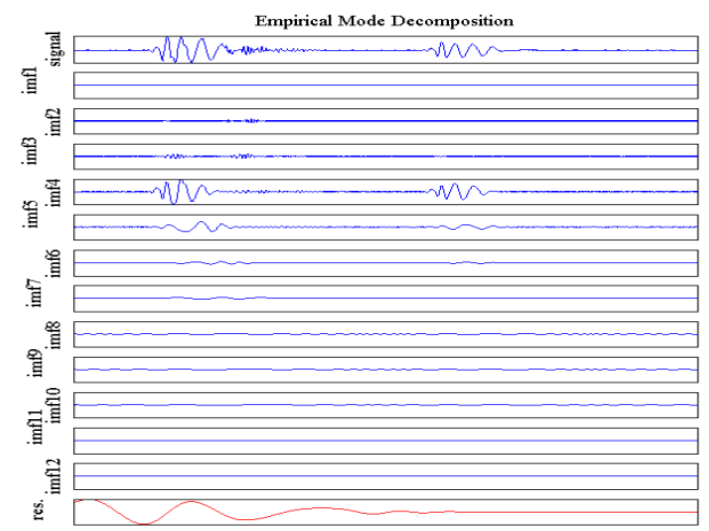

Fig. 1. EMD of the early aortic stenosis signal: from top to bottom the signal, twelve IMFs and the residue. 


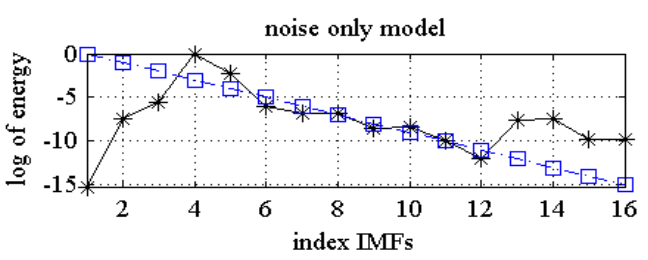

Fig. 2. IMFs selection for early aortic stenosis signal segmentation

(a) original signal

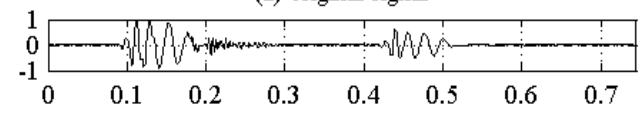

(b) separated signal without murmur

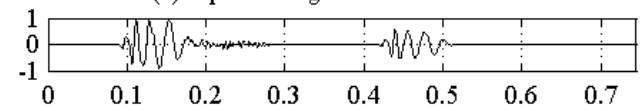

(c) separated murmur

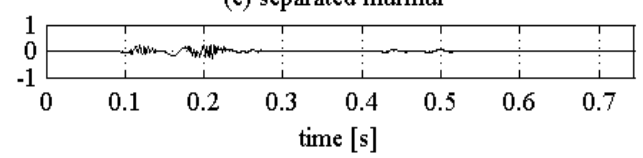

Fig. 3. Segmentation results (a) original signal of early aortic stenosis, (b) separated signal without murmur and (c) pathological murmur.

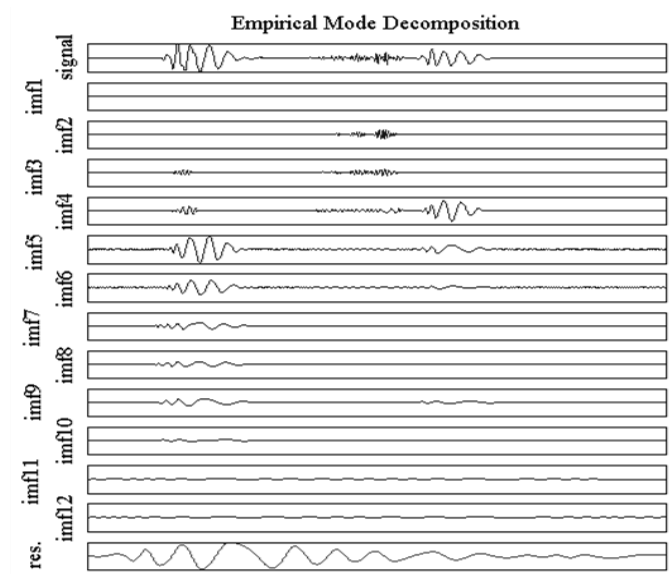

Fig. 4. EMD of the late aortic stenosis signal: from top to bottom the signal, twelve IMFs and the residue.

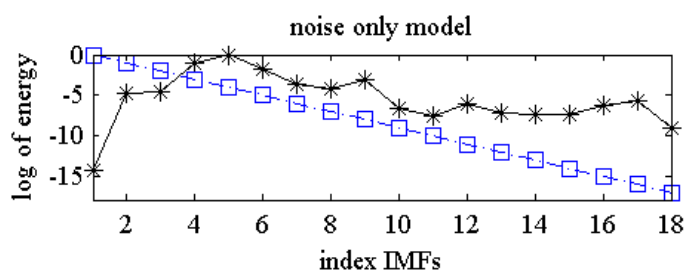

Fig. 5. IMFs selection for late aortic stenosis signal segmentation.

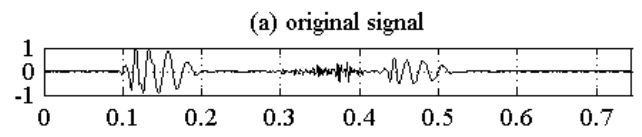

(b) separated signal without murmur

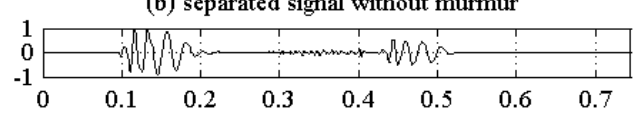

(c) separated murmur

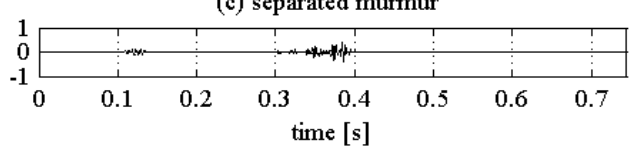

Fig. 6. Segmentation results (a) original signal of late aortic stenosis (b) separated signal without murmur and (c) pathological murmur.

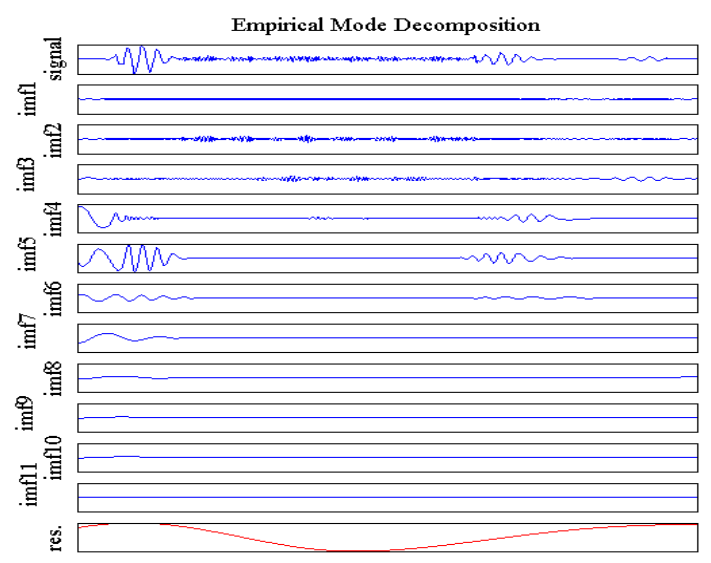

Fig. 7. EMD of the mitral regurgitation signal: from top to bottom the signal, nine IMFs and the residue.

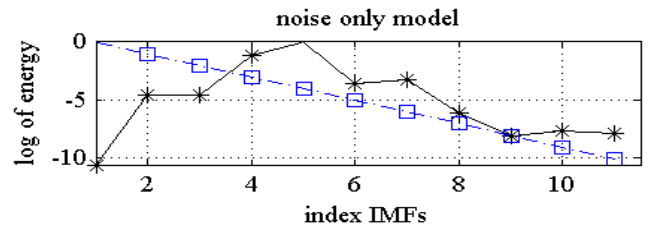

Fig. 8. IMFs selection for mitral regurgitation signal segmentation.

(a) original signal

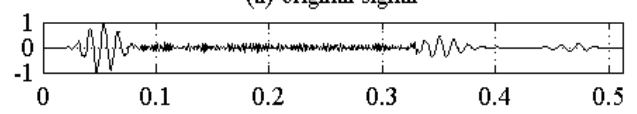

(b) separated signal without murmur

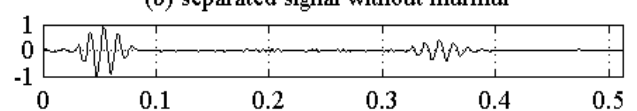

(c) separated murmur

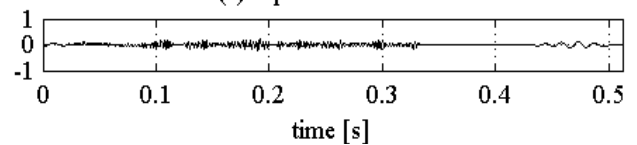

Fig. 9. Segmentation results (a) original signal of mitral regurgitation (b) separated signal without murmur and (c) pathological murmur.

\section{Example 4: Case of Aortic Regurgitation}

The fourth pathological HS signal studied is the Aortic Regurgitation (AR) shown at the top of the Fig. 10. In AR a diastolic murmur is close to the second HS signal S2. It is caused by the backflow of blood across the aortic valve. Depending on the severity of the regurgitation, the murmur may be loud or soft.EMD analysis of the AR signal has given 15 IMFs and a residue in the Fig. 10 where only 12 IMFs have been presented for practical illustration. We can see in this case that only the first two IMFs contain the murmurs signal and the third IMF (IMF3) seems present the start of the main components existing in the original signal. The selected IMFs for HS signal segmentation obtained by the method are illustrated in the Fig. 11. The separated signal is obtained by summing all extracted IMFs greater than the threshold showed in Fig. 12 (b). However the murmurs signal is obtained by summing the IMFs less than the threshold such as illustrated in Fig. 12 (c). 


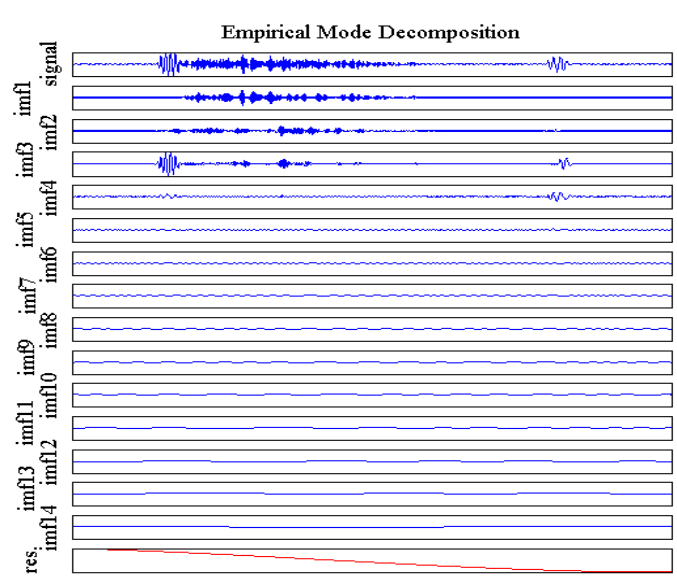

Fig. 10. EMD of the aortic regurgitation signal: from top to bottom the signal, twelve IMFs and the residue.

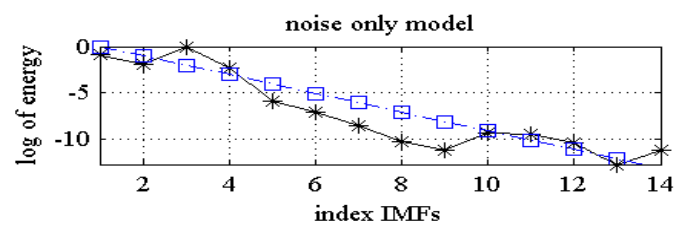

Fig. 11. IMFs selection for aortic regurgitation signal segmentation.

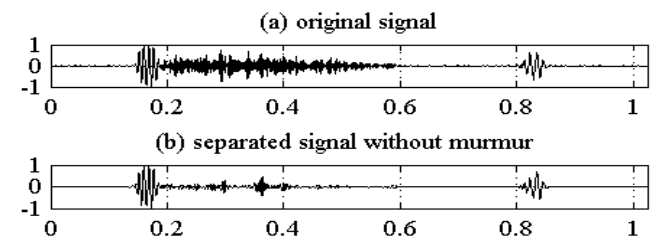

(c) separated murmur

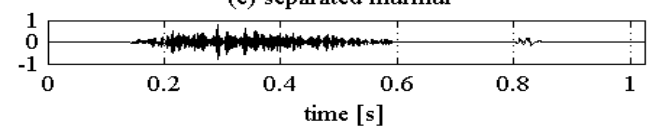

Fig. 12. Segmentation results (a) original signal of aortic regurgitation (b) separated signal without murmur and (c) pathological murmur.

\section{CONCLUSION}

In this paper we proposed the segmentation approaches based on empirical mode decomposition. The method uses the EMD and the noise only model. The experimental results conducted on real life pathological heart sound signals revealed the performance of the proposed methods for segmentation. The noise only model method in conjunction with EMD analysis may be used to discriminate between the IMFs giving the murmur signal and the signal containing the main components. This method is used for the first time in heart sound segmentation. Future work deal with the improvement and extension to a great number an abnormal PCG. In addition, time-frequency study will be added in order to characterise several pathological murmur.

\section{REFERENCES}

[1] N. E. Huang, Z. Shen, S. R. Long, M. C. Wu, H. H. Shih, Q. Zheng, N. Ch. Yen, C. C. Tung, and H. H. Liu, "The empirical mode decomposition and the Hilbert spectrum for nonlinear and non-stationary time series analysis," in Proc. of the Royal Society of London, vol. 454, pp. 903-995, 1998.
[2] G. Rilling, P. Flandrin, and P. Goncalves, "On empirical mode decomposition and its algorithms," IEEE-EURASIP Workshop on Nonlinear Signal and Image Processing - 03, Grado (I), June 2003.

[3] A. O. Boudraa and J. C. Cexus, "Denoising via empirical mode decomposition," in Proc. of IEEE International symposium on control, communications and signal processing, ISCCSP 2006.

[4] Y. Kopsisnis and S. McLauglin, "Empirical mode decomposition based soft thresholding," in Proc. of the $16^{\text {th }}$ European Signal Processing Conference, pp. 43-47, 2008.

[5] O. G. I. Erer and M. Kapaln, "Empirical mode decomposition based denoising for high resolution direction of arrival estimation," in proc of the $17^{\text {th }}$ European signal conference processing, Glasgow Scotland, August 24-28, 2009.

[6] D. Boutana, M. Benidir, and B. Barkat, "Denoising and characterisation of heart sounds signal using optimal mode functions," presented at the $4^{\text {th }}$ international symposium on applied sciences in biomedical and communication technologies, Barcelona-Spain, Oct. pp. 26-29, 2011.

[7] H. Liang, S. Lukkarinen, and I. Hartimo, "A heart sound segmentation algorithm using wavelet decomposition and reconstruction," in Proc. of the IEEE EMBS, $19^{\text {th }}$ annual international conf. 1997, pp. 1630-11633.

[8] D. Kumar, P. Carvalho, M. Antunes, J. Henriques, L. Eugenio, R. Schmidt, and J. Habetha, "Detection of S1 and S2 heart sounds by high frequency signatures," Engineering in Medicine and Biology Society, EMBS '06. $28^{\text {th }}$ Annual International Conference of the IEEE Aug. 30 2006-Sept. 3 , pp. $1410-1416$.

[9] S. Choi, Y. Shin, and H.-K. Park, "Selection of wavelet packet measures for insufficiency murmur identification," Expert System with Applications: An International Journal, vol. 38, no. 4, pp. 4264-4271, April, 2011.

[10] C. X. Feng, Y. MA, C. LIU, X. J. Zhang, and Y. F. Guo, "Research on heart sound identification technology," Science China Press and Springer-Verlag, vol. 55, no. 2, pp. 281-292, Berlin Heidelberg 2011.

[11] D. Boutana, M. Benidir, and B. Barkat, "Segmentation and identification of some pathological phonocardiogram signals using time-frequency analysis," Signal Processing, IET, vol. 5 , no. 6, pp. $527-537,2011$.

[12] Z. H. Wu and N. Huang, "A study of the characteristics of white noise using the empirical mode decomposition method," in Proc. of the Royal Society of London, vol. 460, pp. 1597-1611, June 2004.

[13] P. Flandrin, P. Goncalves, and G. Rilling, "Detrending and denoising with the empirical mode decompositions," in proc. of the European Signal Processing Conference, 2004, pp. 1582-1584.

[14] Egeneralmedical. [Online].

Available: http://www.egeneralmedical.com/.

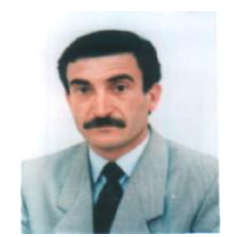

Daoud Boutana received his high study degree in Physics and Magister from University of Bab Ezouar in Algiers in 1982 and 1986. From September 1989 to 2005 he worked as an assistant lecturer in the department of automatic and electronic, university of jijel. He obtained the state Doctorate Degrees in electronic (signal processing) from the department of electronics, Faculty of engineer Science, University of Constantine, Algeria. He effect courses (one month) improvement as visitor during (2003, 2004 and 2005) at the Signal and System laboratory in the University of Paris-Sud Supelec, France. Actually, he is Professor at the department of automatic University of Jijel, Algeria. His main research efforts are focused on the time-frequency signal processing, wavelet analysis with application to speech signal and biomedical signal (PCG, ECG and EEG).

Braham Barkat received the "Ingenieur d'Etat" degree in electronics in 1985 from the National Polytechnic Institute of Algiers (ENPA), Algeria, the M.S. degree in control systems in 1988 from the University of Colorado, Boulder, and the Ph.D. degree in signal processing in 2000 from the Queensland University of Technology (QUT), Brisbane, Australia. His research interests include time-frequency signal analysis, estimation \& detection, and statistical array processing.

Messaoud Benidir received the Engineering degree from the central school of Paris option applied mathematics in 1975.He is Professor at the University Paris XI - Orsay and graduate school of electricity, signal and system laboratory. His research interests include time-frequency signal analysis, and statistical array processing. 